\title{
Linx
}

Revue des linguistes de l'université Paris X Nanterre

$8 \mid 1996$

Du dire et du discours

\section{La construction de dialogues télématiques : quelques stratégies discursives}

Jacques Anis

\section{(2) OpenEdition}

\section{Journals}

Édition électronique

URL : http://journals.openedition.org/linx/1165

DOI : 10.4000/linx.1165

ISSN : 2118-9692

\section{Éditeur}

Presses universitaires de Paris Nanterre

\section{Édition imprimée}

Date de publication : 1 septembre 1996

Pagination : 173-185

ISSN : 0246-8743

\section{Référence électronique}

Jacques Anis, «La construction de dialogues télématiques : quelques stratégies discursives », Linx [En ligne], 8 | 1996, mis en ligne le 13 juillet 2012, consulté le 02 mai 2019. URL : http:// journals.openedition.org/linx/1165; DOI : 10.4000/linx.1165

Ce document a été généré automatiquement le 2 mai 2019.

Département de Sciences du langage, Université Paris Ouest 


\title{
La construction de dialogues télématiques : quelques stratégies discursives
}

\author{
Jacques Anis
}

1 Cet article n'est pas le premier que nous inspire la communication télématique, à laquelle nous nous intéressons depuis dix ans ${ }^{1}$. Nous avons cependant, dans le cadre d'un ouvrage à paraître, Écrit, écriture, ordinateur, recueilli de nouveaux corpus, qui nous ont permis d'approfondir notre exploration. Un article paru dans LINX (Anis 1994) traite de la ponctuation des dialogues. Ici, conformément à la thématique de ce numéro d'hommage à Denise Maldidier, nous porterons notre regard sur la construction discursive. ${ }^{2}$

\section{Le dialogue télématique ${ }^{3}$}

2 Les "messageries anonymes"4 du réseau Télétel permettent à des personnes de dialoguer librement sous couvert d'un pseudonyme. La plupart sont consacrées à un érotisme débridé. Un très petit nombre, que l'on peut qualifier de "conviviales", abordent des sujets plus variés et l'érotisme y est moins direct. C'est le cas de la messagerie Bistro ${ }^{5}$, sur laquelle nous avons enregistré 14 dialogues (entre nous-même, sous quatre pseudonymes différents, et 14 interlocuteurs différents ${ }^{6}$ ). La plupart des usagers sont des habitués, et rendent publiques des CV (cartes de visite), par lesquelles ils se présentent dans un style parfois réaliste, mais le plus souvent ludique (voir Annexe 2).

\section{Une conversation}

3 Il s'agit d'échanges non institutionnels, dont les buts ne sont pas strictement définis. En règle générale, il n'y a pas de relation sociale préalable entre les partenaires. Les thèmes abordés ne sont pas prévus d'avance. Cependant, les échanges sont conditionnés par une finalité générale - construire une relation positive, pouvant éventuellement déboucher sur une rencontre -, et ils se conforment à un modèle général - ils comportent en règle 
générale une attaque fondée sur le pseudo ou la CV (voir Annexe 2), puis une suite de questions/réponses visant à faire apparaître des affinités, et enfin une conclusion fortement ritualisée.

\section{Une conversation écrite, un dialogue} seulement permettent au dialogue d'avancer, mais font émerger des affinités. L'établissement de consensus fondés sur les points communs a donc une importance primordiale. Mais la polémique est aussi un ressort utile, car elle permet à chacun de s'affirmer et se faire valoir; elle est susceptible de générer des consensus de second niveau. Cette polémique relève essentiellement d'un jeu verbal, qui recourt aux mots d'esprit et aux clichés et stéréotypes. Quant à la philosophie de comptoir, elle permet de faire perdurer le dialogue.

\section{Le "moi aussi"}

La recherche d'affinités étant comme on l'a dit un moteur du dialogue, on ne s'étonnera pas qu'un axe essentiel soit "moi je... moi aussi", qui fait, dans le meilleur des cas, émerger un "nous". La découverte des affinités est souvent marquée de manifestations de joie.

\begin{tabular}{|l|l|}
\hline A8 & c'estvrai? figure toi que j'en fais et que j'ecris sur ce sujet \\
\hline Chr9 & c'est génial ca \\
\hline
\end{tabular}


ou quand isa et AMATEUR communient dans le culte de Verlaine :

\begin{tabular}{|l|l|}
\hline A18 & je te suis tt a fait j'aime beaucoup verlaine il est poignant \\
\hline Is18 & ha je suis contente
\end{tabular}

ou encore quand Death is a dialogue et AMATEUR se découvrent des affinités idéologiques (Death a demandé à son partenaire de se présenter à nouveau) :

\begin{tabular}{|l|l|}
\hline A20 & jacques 41 a athee et fier de l'etre \\
\hline De20 & $\begin{array}{l}\text { Hahahaa } \\
\text { je suis athee aussi voire agnostique! }\end{array}$ \\
\hline
\end{tabular}

10 On remarquera le désir de surenchère marqué par le "voire" (bien que dans l'usage habituel, la hiérarchie soit plutôt inverse). Une telle surenchère pourrait être ambiguë, car, si sur un fond commun, l'un prétend pousser plus loin les choses, il semble revendiquer une supériorité sur l'autre.

11 De fait le "moi aussi" peut être paradoxalement dissensuel. On observera un glissement du positif au négatif dans ce passage qui inaugure le dialogue entre Ann O'Mally et lotophage :

\begin{tabular}{|l|l|}
\hline An1 & mais z'avez lu homere \\
\hline L1 & eh oui dans ma jeunesse \\
\hline \hline An2 & comme nous tous quoi \\
\hline L2 & j'ai fait du grec jadis \\
\hline An3 & grec ou non l'odysee etait au programme \\
\hline
\end{tabular}

12 On peut constater que la deuxième réplique de lotophage, qui revendique une relation spécifique à l'Odyssée, irrite son interlocutrice, qui renvoie cette lecture au bagage culturel commun fourni par l'institution scolaire ("au programme").

Les effets positifs du "moi aussi" peuvent être annihilés par une démarche réductrice, comme en témoigne cet extrait :

\begin{tabular}{|l|l|}
\hline F17 & vous faites quoi dans la vie \\
\hline Di18 & informatique... (genial non ??) \\
\hline F18 & tout le monde en fait de l'informatique \\
\hline Di19 & Ah bon vous aussi ? \\
\hline
\end{tabular}




\begin{tabular}{|l|l|}
\hline F19 & entre autres \\
\hline Di20 & Oh mon petit quel bonheur nous avons des points communs \\
\hline F20 & celui la n'est guere pointu \\
\hline
\end{tabular}

Comme on le voit, Dionysos commence par mettre en avant sa spécialité; lafemmeillustree lui oppose un "tout le monde en fait..." qui ravale ses prétentions. Il cherche à récupérer néanmoins le "point commun" au service de la construction du "nous", mais est "rembarré" par son interlocutrice.

15 À l'inverse du "moi aussi" dissensuel, on trouvera le "pas moi" consensuel. Il s'agit d'accepter l'autre dans sa différence et de lui en donner acte.

\begin{tabular}{|l|l|}
\hline L13 & \multicolumn{1}{|c|}{ tu es de quel coin } \\
\hline \hline An14 & colmar en exil a paris \\
\hline L14 & moi j'ai toujours vecu en rp paris puis proche banlieue \\
\hline An15 & je suisla depuis pas mal de temps mais j'aime pas rop \\
\hline \hline L15 & moi j'aurais peur de m'ennuyer en province \\
\hline An16 & moi non mais j'y ai ete elevee. et puis ca depend ou \\
\hline
\end{tabular}

La dernière réplique prend acte de la différence, la relativise ("mais") par une explication biographique, et concède enfin qu'on puisse s'ennuyer dans certains sites provinciaux ${ }^{9}$.

\section{Polémiques}

La polémique a l'intérêt de faire rebondir le dialogue, de permettre aux interlocuteurs de briller, de se reconnaitre mutuellement comme brillants, de susciter des consensus de second degré.

La polémique peut surgir quand l'un évalue la démarche de l'autre, qui se défend :

\begin{tabular}{|l|l|}
\hline Lg4 & sourire..c'est rare comme pseu \\
\hline L4 & il faut essayer de surprendre \\
\hline Lg5 & oui..mais la..je doute que la surprise soit tres comprehensible \\
\hline L5 & pas grave les gens se posent des questions au moins \\
\hline Lg & sourire..oui..c'est deja ca \\
\hline
\end{tabular}


19 On notera les procédés caractéristiques d'une discussion polie. L'expression du désaccord (Lg5) est précédée d'un "oui" concessif, suivi d'un "mais là" qui indique la divergence de vue, présenté sous la forme atténuée d'un "je doute que...". Lotophage accepte implicitement la pertinence de l'argument, qu'il se contente de relativiser, puis de détourner à son profit, assez modestement toutefois : "au moins". Cette fois, le partenaire acquiesce sans réserve et son "c'est déjà ça" fait écho à "au moins".

La polémique prend parfois la forme d'une joute oratoire. AMATEUR attaque le dialogue avec une certaine agressivité, qui répond au ton provoquant de la CV d'isa (voir Annexe 2).

\begin{tabular}{|l|l|}
\hline A1 & votre cv est une vraie profession de foi vs y croyez? \\
\hline Is1 & profession de foi? dis pas de grots mots stp \\
\hline A2 & je veux dire un vrai laius electoral \\
\hline Is2 & tu persistes? \\
\hline A3 & bref un truc au nieme degre pour que si on croit avoir compris on se croit au top \\
\hline \hline Is3 & ca s'est un peu vrai par contre \\
\hline A4 & ah enfin j'ai marque un point a maligne malin et demi! \\
\hline Is4 & c ton but marquer des points sur des connes? \\
\hline A5 & $\begin{array}{l}\text { la t'exageres je te prends pas du tout pour une conne je pense meme que t'es moins conne } \\
\text { que beaucoup }\end{array}$ \\
\hline Is5 & ce qui importe c'est ce que je pense ms j'aime exagerer. qui es tu? \\
\hline
\end{tabular}

21 La question initiale d'AMATEUR met en cause la sincérité de la CV, jouant sur la contradiction entre "profession de foi" et non-croyance. C'est le genre de question qui n'appelle pas une vraie réponse. Isa choisit de rejeter l'assertion qui précédait la question, mais par une stratégie métadiscursive : cette assertion serait injurieuse, car contenant des "gros mots", évaluation qui ne peut se fonder que sur le refus d'un cadre idéologique qui reste implicite, celui de la religion. Quand AMATEUR propose le terme "laïus électoral", métaphore qui relève d'un autre domaine cognitif, mais suggère encore plus une rhétorique non sincère, la réplique "tu persistes ?" indique de manière extrêmement concise que la politique a pour isa le même statut que la religion et elle témoigne de la même résistance teigneuse au dialogue que la réplique précédente. On remarquera la différence stylistique entre les deux partenaires, le tutoiement et le registre plus familier d'isa suggérant une plus grande violence. Le "bref" qui ouvre la réplique d'AMATEUR est de nature dialogique : finissons-en avec le débat sur la forme. L'emploi du terme "truc" (qui, de par sa signification hautement schématique ${ }^{10}$ contraste avec "profession de foi" et "laïus électoral") est de ce point de vue significatif, car il serait difficile de le rattacher à un cadre idéologique! Il s'inscrit aussi dans un style familier ("nième degré", "au top"), qui suggère une espèce d'énervement ou d'excitation ${ }^{11}$. L'interlocutrice accepte ce 
jugement, de manière nuancée - "un peu vrai" -, et le "par contre", s'il montre qu'elle reste sur ses positions pour ce qui précède, souligne le tournant dans le dialogue. AMATEUR n'adopte pas pour autant une attitude consensuelle, exprimant son agressivité dans la métaphore sportive "marquer un point" et par le cliché "à $\mathrm{X}, \mathrm{X}$ et demi". Isa réagit assez subtilement, en généralisant : le pluriel "des connes" semble déteindre sur l'énoncé tout entier, le présent devient alors générique et "c'est ton but" est compris comme "ton but dans la vie". A une telle question, on ne peut que répondre "non", ce serait donc inutile, d'autant plus que le terme des "connes" a été placé là (avec le statut de présupposé) comme une "perche". Quand quelqu'un s'applique à soi-même le terme en question, on ne peut l'interpréter au premier degré ; en l'occurrence, "des connes" relève de la polyphonie énonciative et peut être paraphrasé par "des femmes que tu considères comme des...". Telle est la lecture d'AMATEUR, qui réagit par la protestation polie qu'on attendait de lui : "là t'exagères je ne te prends pas du tout pour une conne". Dans la deuxième partie de sa réponse, il tente d'aller plus loin "même" pour esquisser un compliment, qui semble gauche, car être "moins c... que beaucoup", présuppose quand même une certaine dose de c...rie". Heureusement, isa ne tient compte que de l'orientation globale de cette intervention - le passage d'AMATEUR au tutoiement vise sans doute à améliorer le contact - et, après avoir concédé un goût pour l'exagération dont la $\mathrm{CV}$ et ce début de dialogue témoignent, elle prend l'initiative d'un nouveau départ, qu'on pourra d'ailleurs juger exagérément plat: "qui es-tu?". Tout se passe comme si l'interlocuteur avait réussi un test préliminaire, qui le qualifie pour un dialogue.

Assez souvent, la discussion a comme point de départ une contestation du "nous". Quand la communauté de vue postulée a des bases trop fragiles - interprétation du pseudo, idées générales - il peut se créer une fracture. Réagissant au pseudo "Death is a Dialogue", AMATEUR s'oriente sur une fausse piste :

\begin{tabular}{|l|l|}
\hline A2 & et s'il n'y a que poussiere.. \\
\hline De2 & c'est vach'ment triste \\
\hline A3 & reste plus qu'à se saouler! \\
\hline De3 & je ne me saoule plus \\
je ne supporte plus les gueules de bois! \\
\hline A4 & et tu arrives à te supporter? \\
\hline De4 & $\begin{array}{l}\text { oui tres bien } \\
\text { pourquoi tu as des pb? }\end{array}$ \\
\hline A5 & non mais la condition humaine... \\
\hline De5 & je suis eperdemente optimiste \\
\hline A6 & avec un tel pseu?? \\
\hline De6 & sourire c'est un poeme d'emily dickenson \\
\hline
\end{tabular}




\begin{tabular}{|l|l|}
\hline A7 & ah bon c au second degre alors \\
\hline De7 & $\begin{array}{l}\text { pas vraiment } \\
\text { je le trouve joli }\end{array}$ \\
\hline A8 & tu es prof d'anglais \\
\hline De8 & non je suis infirmiere smile! \\
\hline
\end{tabular}

Comme on le voit, AMATEUR s'est installé dans les propos de bistrot sur le malheur du monde et croit opportun de suggérer que sans alcool, il est difficile de s'accepter (A4). Le "oui très bien" de Death n'est évidemment pas la réponse attendue et de fait, elle abandonne brutalement le terrain du cliché pour celui de la psychologie personnelle. AMATEUR est réduit à la défensive "non mais", car il ne peut accepter le rôle de celui qui "a des problèmes", il faut qu'il revienne au terrain initial. Mais au prix de se contredire, Death change de philosophie, et AMATEUR n'a plus que le pseudonyme pour tenter de la mettre en difficulté. Malheureusement, Death rejette celui-ci à la pure littérature. AMATEUR n'a d'autre recours que de s'accrocher au thème de l'anglais, mais cela le mène à une fausse hypothèse. Heureusement pour lui, Death va ensuite répondre aux questions sur le métier, qui permettront la poursuite du dialogue.

Dans un autre dialogue, AMATEUR avait mieux réussi à rétablir le consensus :

\begin{tabular}{|l|l|}
\hline A9 & nous sommes de passage et pas sages \\
\hline Li9 & Parlez pour vous..que savez-vous de ma sagesse..? \\
\hline A10 & Si vous etiez sage vous ne seriez pas la \\
\hline Li10 & Oula...vous aimez les chemins de traverse,vous.. \\
\hline A11 & et une insolente ne peut etre sage \\
\hline Li11 & je vous felicite pour toutes vos deductions judicieuses.. \\
\hline A12 & la logique est le seul moyen de delirer \\
\hline Li12 & En l'occurrence l'apparence de la logique fait encore mieux l'affaire. \\
\hline
\end{tabular}

AMATEUR fait surgir d'un jeu de mots une assertion qu'il applique à un "nous". Le référent du pronom personnel est flou, il peut s'agir de l'humanité en général ou des deux partenaires. L'insolente rejetant pour elle-même le jugement de non sagesse, AMATEUR met en œuvre deux arguments a contrario : l'incompatibilité entre sagesse et utilisation du Minitel, et entre sagesse et insolence. L'insolente accepte le verdict de la logique, qu'elle apprécie comme mécanisme purement verbal. D'où un consensus nuancé, l'un avançant un paradoxe sur la logique auxiliaire de la folie, l'autre parlant d'une "apparence" de logique. Mais on débouche sur une harmonie, L'insolente ayant "félicité" 
son interlocuteur et loué son esprit d'à-propos: "En l'occurrence... encore mieux l'affaire".

\section{Le café du Commerce et les trous de la conversation}

Les thèmes généraux sont souvent mobilisés, soit pour quitter le terrain interpersonnel si on n'est pas en position de force, soit pour alimenter la conversation. Le méta-thème de la communication est tout à fait indiqué à cet effet, mais il a ses limites, comme le montrera le passage assez long que nous allons étudier. D'Arabesque ayant utilisé deux fois le procédé usuel de la simulation de cette manifestation para-verbale qu'est le rire (5 : "rire", 6 : "rires"), lafemmeillustree termine son intervention 6 par "je trouve que vous riez bien facilement" (on notera la connotation autonymique que prend ici "riez"). Suivent les développements ci-dessous :

\begin{tabular}{|c|c|}
\hline Da7 & $\begin{array}{l}\text { Il y a mimmme facons de rires. } \\
\text { Sur mmntl cela ne se voit pas. } \\
\text { Ce n'est pas le rire qui est d'importanc } \\
\text { e 'cest la facon. }\end{array}$ \\
\hline F7 & et qu'elle est votre facon? \\
\hline Da8 & $\begin{array}{l}\text { Mais sur mntl ca ne peut pas s'expliquer } \\
\text {..Les mmots ne peuvent tout decrire }\end{array}$ \\
\hline F8 & bien vu digne de nietzsche! \\
\hline Da9 & $\begin{array}{l}\text { La philosophie de comptoir ne m'interess } \\
\text { e pas. } \\
\text { Non plus que zarahoustra d'ailleurs. }\end{array}$ \\
\hline F9 & la philosophie de bistro pourtant semble vous plaire \\
\hline Da10 & mais oui. \\
\hline F10 & a part ca? \\
\hline Da11 & he oui que dire desormais? \\
\hline F11 & c'est la question \\
\hline Da12 & $\begin{array}{l}\text { C'est le silence, enfin si l'on peut di } \\
\text { re }\end{array}$ \\
\hline F12 & le silence se meuble \\
\hline Da13 & $\begin{array}{l}\text { Un silence sur mntl ca n'a pas } \\
\text { d'importance en fait. }\end{array}$ \\
\hline F13 & rien n'a d'importance ici \\
\hline
\end{tabular}




\begin{tabular}{|l|l|}
\hline Da14 & Ca c'est moins sur. \\
\hline F14 & rien ne compte a part le non dit \\
\hline Da15 & $\begin{array}{l}\text { C'est quoi le non dit ici? } \\
\text { Il se niche ou? }\end{array}$ \\
\hline F15 & dans le noir de l'ecran \\
\hline Da16 & Quelle horreur! \\
\hline \hline & Le vide spatial. \\
\hline F16 & le vide absolu \\
\hline \hline Da17 & L'angoisse quoi! \\
\hline F17 & le neant est reposant parfois \\
\hline \hline Da18 & On se repose grace a quelque cchose jamais avec rien. \\
\hline F18 & on pourrait changer de sujet, non? \\
\hline F19 & Mais oui ,comme vous voulez. \\
\hline
\end{tabular}

L'intervention d'Arabesque sur le rire génère une sorte d'aporie, s'il est vrai que ce qui compte c'est la "façon" de rire et qu'elle ne peut se manifester sur minitel. D'où le lieu commun "les mots ne peuvent tout décrire". À la suite de la raillerie sur Nietzsche, d'Arabesque s'enferre quelque peu à rejeter une "philosophie de comptoir" qu'il est précisément en train de pratiquer sous sa forme télématique "philosophie de <la messagerie> bistro". Cette contradiction que fait apparaitre lafemmeillustree ("pourtant"), il doit la reconnaître, par un laconique "mais oui", qui provoque un blocage. Les deux interventions suivantes se contentent d'en prendre acte. L'invite de lafemmeillustree "à part ça ?" ne reçoit pas de réponse, l'autre se contente d'en confirmer la pertinence ("hé oui") et d'expliciter la situation: "que dire désormais ?"). Nouvelle intervention de pur commentaire "c'est la question", puis glissement ${ }^{12}$ vers la thématisation du blocage comme "silence". La réplique F12 est ambiguë, on peut la lire comme générique ou en situation, et du point de vue modal comme potentielle ou déontique. Da13 respécifie le nom "silence", dans la situation particulière de la communication télématique (d'où la locution adverbiale "en fait"), et en dénie l'importance. F13 élargit l'insignifiance à la communication télématique dans sa globalité. Da14 marque un écart par rapport au discours de sa partenaire ("ça c'est moins sûr"). Cette dernière continue dans le style philosophie creuse. D'Arabesque formule une question à caractère polémique, comme le montre l'attaque "C'est quoi...", question qu'il reformule de manière plus aiguë en la métaphorisant spatialement (spatialisation préparée par la désignation "ici") et en utilisant un style parlé (construction et verbe "se nicher"). Lafemmeillustree répond à cette question dans le cadre de la communication télématique et d'Arabesque s'appuie sur cette réponse pour développer un discours spléenétique. On s'enfonce dans le négatif, malgré un essai de lafemmeillustree en F13: 
prédication d'un attribut positif au néant, modalisée par l'adverbe parfois. Mais le partenaire repousse avec énergie la proposition: opposition entre "quelque chose" (introduit par le positif "grâce à") et "rien", utilisation de "jamais", construction antithétique de l'énoncé en deux segments (avec double espace entre les deux). Il ne reste plus à la femmeillustree, compte tenu de l'attitude non coopérative de son interlocuteur, qu'à prendre en charge explicitement la gestion du dialogue en proposant un changement de sujet, ce à quoi l'autre consent, mais avec une certaine mollesse : de nouveau un "mais oui", qui laisse à la charge du partenaire de trouver un nouveau thème. Il ne restera d'autre issue à celle-ci que de questionner d'Arabesque sur son pseudo.

\section{BIBLIOGRAPHIE}

ANIS, J. (1987) Le vidéotex interactif : un nouvel espace-temps pour la communication écrite, Anis, J. et Lebrave, J.-L. (éds), LINX 17, Le texte et l'ordinateur, C.R.L. de Paris X Nanterre, pp. 46-55.

-, (1994) Pour une graphématique des usages : le cas de la ponctuation dans la communication télématique, Anis, J. (éd.), LINX 31, Écritures, pp. 81-97.

-, (à par.) Écrit, écriture, ordinateur.

ANIS, J. et LEBRAVE, J.-L (1986) Des textes interactifs ?, en coll. avec Lebrave, J.-L., Filliolet, J. et Galmiche, M. (éds), LINX 14, Langue et machines, C.R.L. de Paris X Nanterre, pp. 107-131

DELAS, D. (1985) Petit lexique dialogal, Le français aujourd'hui 71, pp. 97-104.

LANGACKER, R. (1987) Foundations of Cognitive Grammar, vol. 1, Theoretical Foundations, Stanford U. Press.

-, (1991) Foundations of Cognitive Grammar, vol. 2, Descriptive Application, Stanford U. Press.

LÉVY, E. (1993) Langage et interaction dans une situation conversationnelle médiatisée par Minitel, LINX 28, Centre de Recherches Linguistiques de Paris X Nanterre, pp. 69-84.

\section{ANNEXES}

\section{Annexe 1 : relevé des dialogues}

\begin{tabular}{|l|l|l|l|l|}
\hline $\begin{array}{l}\text { Pseudo de l'Interlocuteur } \\
\text { suivi de notre abréviation }\end{array}$ & $\begin{array}{l}\text { Pseudo de l'Observateur } \\
\text { suivi de notre } \\
\text { abréviation }\end{array}$ & $\begin{array}{l}\text { Nbre } \\
\text { messages } \\
\text { Inter. }\end{array}$ & $\begin{array}{l}\text { Nbre } \\
\text { messages } \\
\text { Obs. }\end{array}$ & $\begin{array}{l}\text { Nbre } \\
\text { messages } \\
\text { total }\end{array}$ \\
\hline juliette1 (Ju) & PASSANT\$13(P) & 11 & 11 & 22 \\
\hline avoinemandchoue (Av) & PASSANT\$ & 9 & 10 & 19 \\
\hline
\end{tabular}




\begin{tabular}{|l|l|l|l|l|}
\hline Ann O'Mally\$(An) & lotophage (L) & 18 & 18 & 36 \\
\hline le gerfaut\$(Lg) & lotophage & 7 & 6 & 13 \\
\hline myrabelle\$ (My) & MACHO (M) & 15 & 15 & 30 \\
\hline Chrystal\$ (Chr) & AMATEUR (A) & 28 & 28 & 56 \\
\hline isa (Is) & AMATEUR\$ & 19 & 19 & 38 \\
\hline L'insolente (Li) & AMATEUR\$ & 19 & 20 & 39 \\
\hline Death is a Dialogue (De) & AMATEUR\$ & 33 & 33 & 66 \\
\hline canelle\$ (Ca) & AMATEUR & 14 & 14 & 28 \\
\hline \hline Dionysos\$(Di) & lafemmeillustree (F) & 31 & 31 & 62 \\
\hline hombre\$ (Ho) & lafemmeillustree & 12 & 13 & 25 \\
\hline d'Arabesque\$ (Da) & lafemmeillustree & 24 & 24 & 48 \\
\hline tomas\$ (To) & lafemmeillustree & 11 & 11 & 22 \\
\hline \hline & & Total & Total & Total \\
\hline & & 251 & 253 & 504 \\
\hline
\end{tabular}

Annexe 2 : copie d'écran de CV

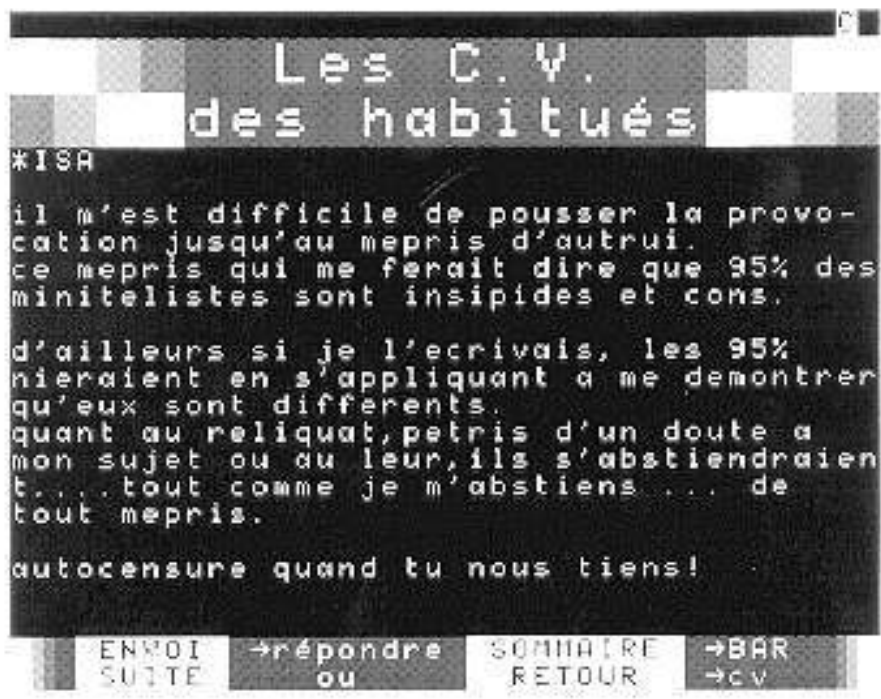




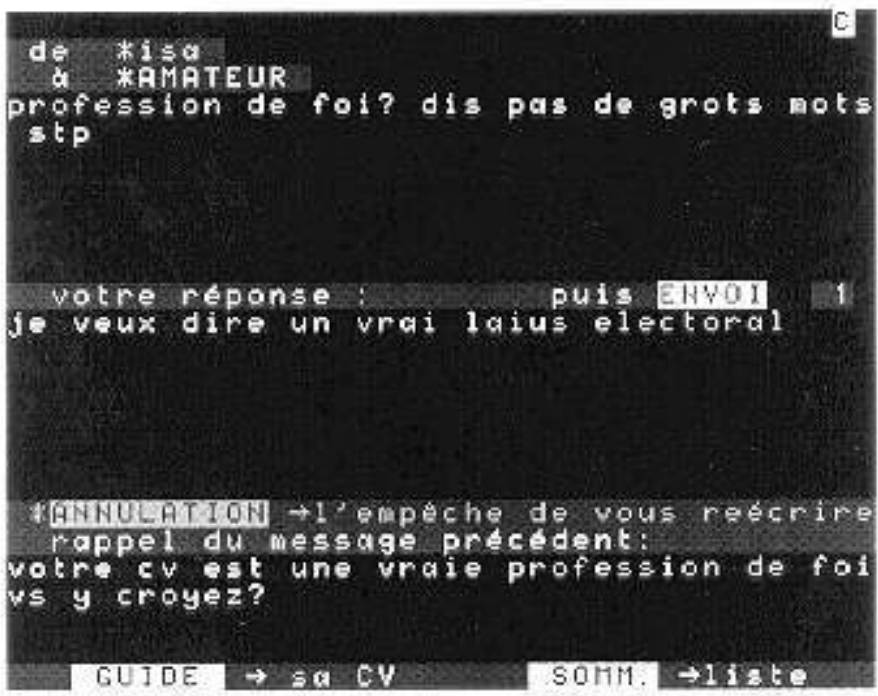

\section{NOTES}

1. Voir notamment Anis et Lebrave (1986) et Anis (1987).

2. Certes, il ne s'agit pas d'analyse du discours au sens étroit du terme, mais on notera que dans Langages $\mathrm{n}^{\circ} 81$, Analyse de discours : nouveaux parcours, D. Maldidier (éd.), 1986, une place était faite à l'analyse conversationnelle (voir notamment Conein, B., Conversation et interaction sociale : analyse de séquences d'offre et d'invitation, pp. 111-120), dont relève en partie cette étude.

3. Nous résumons ici des éléments qui sont beaucoup plus développés dans Anis (à par.). On pourra se reporter également au travail d'un jeune chercheur, Emmanuelle Lévy, qui a travaillé sur le même terrain que nous (Lévy 1993).

4. C'est ainsi que les désigne la terminologie officielle de France Télécom.

5. Accessible par le 3614 (tarification basse avec réductions heures creuses), grâce à des "yops" acquis par des temps de connexion en 3615 (code d'accès "Bar").

6. Voir tableau en annexe. S'y reporter pour les abréviations.

7. D'après Delas $(1985,98)$.

8. On respecte les graphies non conventionnelles, et les spatialisations déterminées par les limites de l'écran et les options individuelles; les - - - en début d'extrait compensent spatialement les segments non reproduits; nous avons simplement parfois ajouté des italiques de mise en valeur. Les citations en dehors des extraits sont en revanche normalisées graphiquement, pour faciliter la lecture.

9. Une démarche analogue a d'ailleurs été appliquée au thème précédent, Noèl (An13) "en ville oui c'est assommant mais en famille c'est joli".

10. au sens de Langacker (1987, 1991, passim).

11. Cela pourrait peut-être expliquer en partie la faute d'orthographe sur la seconde occurrence du verbe croire - comme celle d'isa au tour suivant : "ça s'est".

12. On passe d'un "c" anaphorique, à un "c" désignant le "cadre abstrait", la situation énonciative en général (voir ce que dit Langacker du "es" allemand, dans certaines constructions, 1991: 351sqq.) et du même coup, le verbe "être" passe d'une valeur identifiante à une valeur phénoménologique (= "se produit", "a lieu"). 


\section{RÉSUMÉS}

Après avoir caractérisé le dialogue télématique des messageries conviviales comme une conversation écrite, nous explorons quelques stratégies utilisées par les partenaires pour faire évoluer positivement le dialogue. Le "moi aussi" marque la découverte d'affinités, mais la polémique fait rebondir le dialogue et débouche sur des consensus de second niveau. Quant à la "philosophie de bistro", elle comble les trous de la conversation.

First I characterize the videotext dialog as a written conversation. Then I describe some strategies which participants use to make the dialog proceed in a felicitous way. The "me too!" sanctions the discovering of affinities, but polemics revives the dialog and raises second level consensus. And to fill conversation gaps "bistro philosophy" is at hand.

\section{AUTEUR}

\section{JACQUES ANIS}

(Paris X - Nanterre)

290 avenue d'Argenteuil

F-92600 ASNIERES

e-mail : anis@u-paris10.fr 